\title{
Bench marks
}

\section{Everyday equipment for routine analysis.}

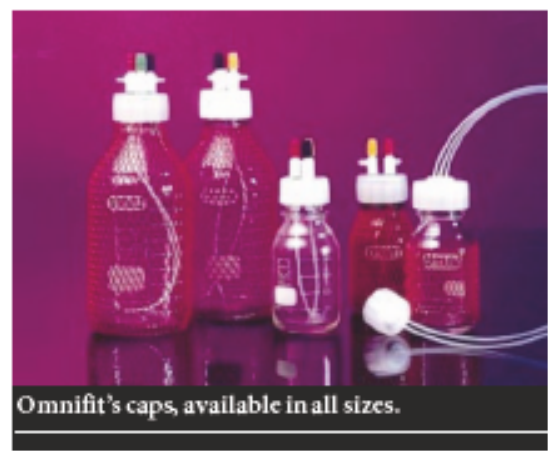

Chemically inert bottle caps

Omnifit

www.omnifit.com

If the cap fits

These chemically inert PTFE bottle caps can be used with aggressive clean-in-place solutions, as well as for connecting tubing to inert fluidic systems, solvent reservoirs or wastebottles. Available in standard GL45 and GL32 thread sizes, with other sizes available on request, the bottle caps come in three basic designs, depending on requirements. A 'dualconnection' design is ideal for applications requiring a variety of tubing types and/or sizes to be connected to a single cap, while the 'no-twist' design has a central independently rotating PTFE block into which tubing lines are connected. The third option, a 'low profile' design ideal for limited space, is supplied fitted with 1-metre lengths of PTFE tubing already connected to the cap.

Microlute

\section{Porvair Sciences}

www.porvair-sciences.com Manifold benefits

Porvair's new acrylic manifold for the Microlute microplate system allows total automation of high-throughput solid phase extraction or protein precipitation experiments. Compatible with most commercial robotic control systems, the manifold permits robotic manipulators torapidly and simply access and lift just the upper part of the Microlute. Each manifold comes complete with an adapter to fit standard $1 \mathrm{ml}$ plates, waste collection reservoir trayand asquarewell collection plate.

Genevac Model DD-4X

Genevac www.genevac.com

General-purpose centrifugal solvent evaporation

Improved speed of evaporation, shorter condenser defrost time, more powerful software (including remote control functions) and the flexibility to accommodate a wide range of sample formats are key benefits claimed for the new Model DD-4X. A redesigned centrifuge chamber, together with powerful IR lamps, dramatically increases the speed of evaporation. To improve high-throughput capability thewaste solvent condenser chamber volume on the DD- $4 \mathrm{X}$ has been raised and the condenser power has been increased by $50 \%$. Interchangeable high-performance, solid aluminium rotors offer high-capacity handling of test tubes and vials. Alternatively, a swing rotor will accommodate tubes, vials, microtitre plates and Fast-Stack multi-deck microplate holders.

\section{Pipetboy Comfort}

Integra Biosciences Group

www.integra-biosciences.com

\section{Lightweight ergonomic pipette}

Weighing only 190 grams, the new Pipetboy Comfort is designed for comfortable singlehanded pipetting in the 1-100 millilitre volume range. The PipetboyComforthasin-built features to prevent accidental misuse. Incorporating a sterile PTFE-membrane filter the
Pipetboy Comfort prevents contamination from overfilling as the penetration of liquid is prevented. In addition the Pipetboy comfort will not squirt out under pressure even if a filled pipette is unintentionally aspirated. For sensitive samples all parts of the Pipetboy Comfort that come into contact with liquid can be autoclaved clean at $121^{\circ} \mathrm{C}$ for $30 \mathrm{~min}$ utes. It also comes with a combined all-in-one stand and charging station that can be wallmounted or can siton the laboratorybench.

These notes are compiled in the Nature office from information provided by the manufacturers. 